Data Repository 2006128

\author{
A High-Resolution Absolute-Dated Indian Monsoon Record Between 53-36 ka \\ from Xiaobailong Cave, Southwestern China \\ Yanjun Cai \\ Zhisheng An \\ State Key Lab of Loess and Quaternary Geology, Institute of Earth Environment, Chinese \\ Academy of Sciences, Xi' an 710075, China
}

Hai Cheng

R. Lawrence Edwards

Megan J. Kelly

Department of Geology and Geophysics, University of Minnesota, MN 55455, USA

\title{
Weiguo Liu
}

State Key Lab of Loess and Quaternary Geology, Institute of Earth Environment, Chinese Academy of Sciences, Xi' an 710075, China

\author{
Xianfeng Wang \\ Department of Geology and Geophysics, University of Minnesota, MN 55455, USA
}

Chuan-Chou Shen

Department of Geosciences, National Taiwan University, Taipei 106, Taiwan, China

\section{Cave and sample descriptions:}

XiaoBailong (Junior Dragon) Cave, 500m long, is located in Mile County, Yunnan, southwestern China. Calcite stalagmite XBL-1 was collected near the cave's end. Present water drip rates in the cave vary between 10-60 drops/minute during the dry season (November to April). However, no modern water dripping was observed at the top of stalagmite XBL-1. Stalagmite XBL-1 is $\sim 59 \mathrm{~cm}$ in height and $15-19 \mathrm{~cm}$ in diameter with a flat center of $4-5 \mathrm{~cm}$ across. The sample is semi-transparent, except for the section 51-55 cm from the top which is light brown in color (Fig. DR1). There is no obvious hiatus in this sample.

\section{Subsampling method and isotope measurement}

Subsamples for stable isotope analysis were collected in two ways, depending on growth rates: 1) drilling with a dental drill of $0.5 \mathrm{~mm}$ diameter directly from the polished half of the stalagmite at an average interval of $2 \mathrm{~mm}$; 2) cutting the stalagmite into a $1 \mathrm{~cm}$ wide slab using a diamond saw and then shaving off the subsamples at a mean resolution $\sim 15$ subsamples per $\mathrm{mm}$. The second method was applied for sections between 100 and $158 \mathrm{~mm}$, and between 445 and $590 \mathrm{~mm}$ from the top, where the growth rates are relatively slow (Figure DR2). We did the stable isotopic analysis on all subsamples collected through the first method and about 1/5 of those by the second one. Oxygen and carbon isotopic compositions of the subsamples were measured on a Finnigan MAT-252 mass spectrometer equipped with Kiel Carbonate Device III in the Institute of Earth Environment, Chinese Academy of Sciences. $\delta^{18} \mathrm{O}$ and $\delta^{13} \mathrm{C}$ values are reported here relative to the Vienna PeeDee Belemnite (VPDB). International standard NBS19 and internal laboratory standard TTB1 were run every 10 to 15 samples and arbitrary selected duplicates were run every 10 to 20 samples. Results show that the precision of $\delta^{18} \mathrm{O}$ analysis is better than $0.15 \%$ o $(2 \sigma)$. 
To test the possible seasonal $\delta^{18} \mathrm{O}$ bias caused by the high resolution sampling (the second method), we compared the $\delta^{18} \mathrm{O}$ profiles from these two sampling methods (15 samples per mm vs. 1 sample per $2 \mathrm{~cm}$ ). The two $\delta^{18} \mathrm{O}$ profiles are essentially the same and demonstrate that the seasonal $\delta^{18} \mathrm{O}$ bias is insignificant (Figure DR3).

Figure DR1. Image of stalagmite XBL-1.

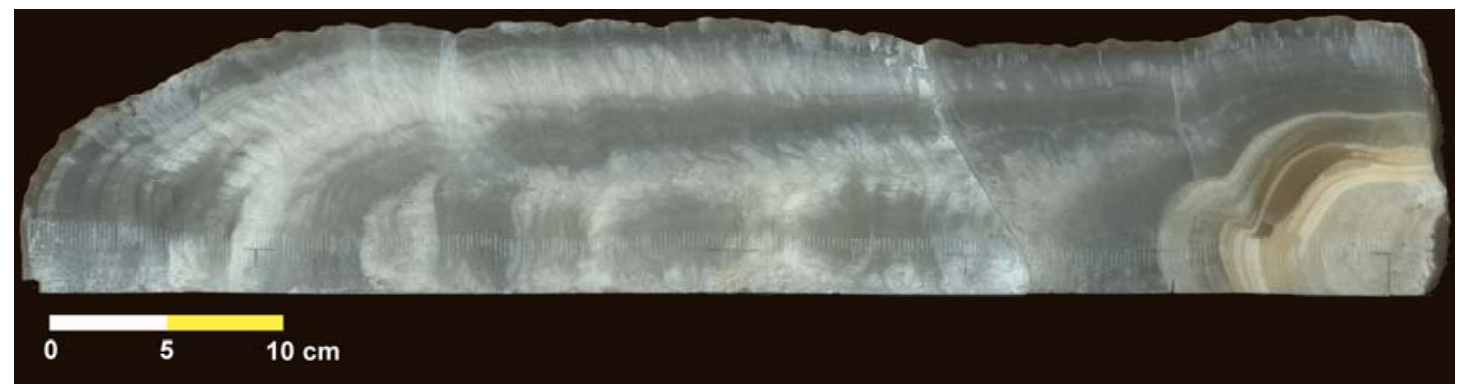

Figure DR2. Plot of age verses depth for stalagmite XBL-1. Error bars indicate ${ }^{230} \mathrm{Th}$ dates and $2 \sigma$ errors. Two vertical gray bars illustrate the portions of XBL- 1 where the shaving method was applied for oxygen and carbon isotope measurements. The drilling method was applied for the rest of the sample.

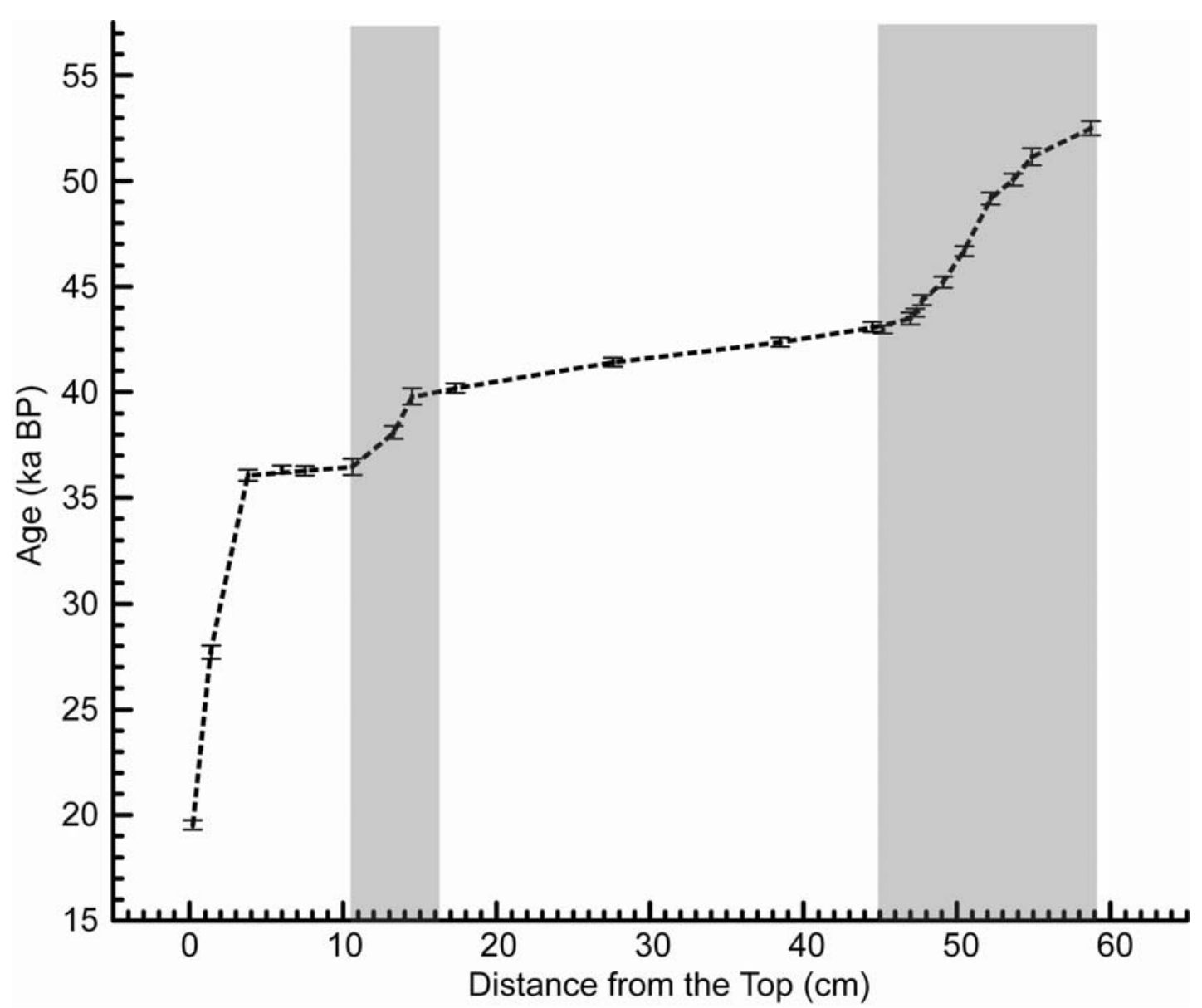


Figure DR3. Comparison of $\delta^{18} \mathrm{O}$ results of subsamples collected by the two different sampling methods. (a) section between 100 and 158mm; (b) section between 445 and $580 \mathrm{~mm}$. Red represents the drilling method while blue represents the shaving method.
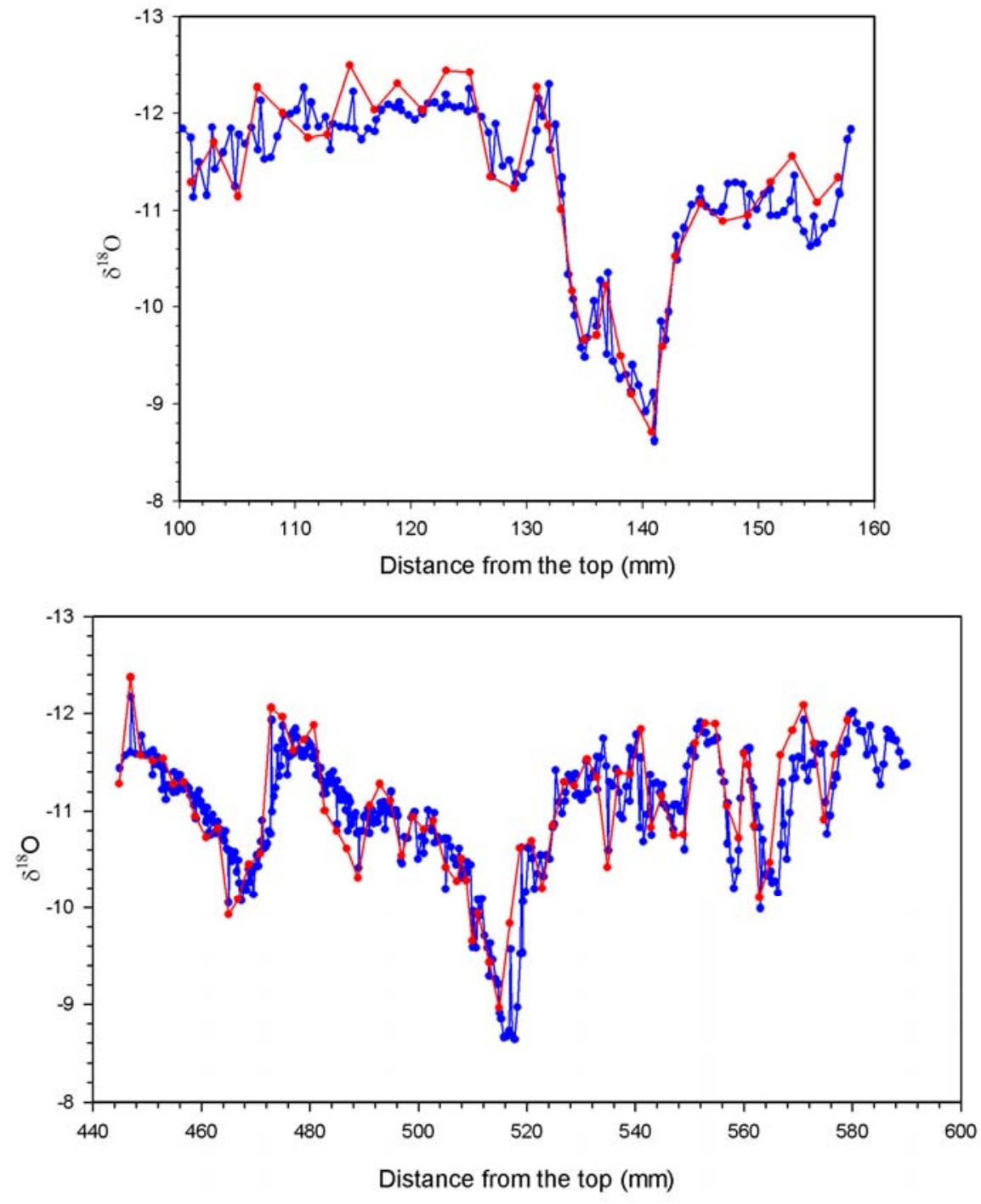
Figure DR4. "Hendy Test" (Hendy, 1971): $\delta^{13} \mathrm{C}$ and $\delta^{18} \mathrm{O}$ measurements along four different growth layers. (a) $\delta^{18} \mathrm{O}$ distributions in four growth layers of stalagmite XBL-1. (b) $\delta^{13} \mathrm{C}-\delta^{18} \mathrm{O}$ correlations in the four layers. Stars denote $\delta^{13} \mathrm{C}-\delta^{18} \mathrm{O}$ data from the layer at $12.8 \mathrm{~cm}$ from the top; triangles $17.9 \mathrm{~cm}$; squares $38.8 \mathrm{~cm}$; and circles $49.9 \mathrm{~cm}$. Three layers $(12.8 \mathrm{~cm}, 17.9 \mathrm{~cm}$ and $49.9 \mathrm{~cm})$ are during interstadials (or the wet phases) and one layer $(38.8 \mathrm{~cm})$ is within the stadial (or the dry phase) portions of the sample.
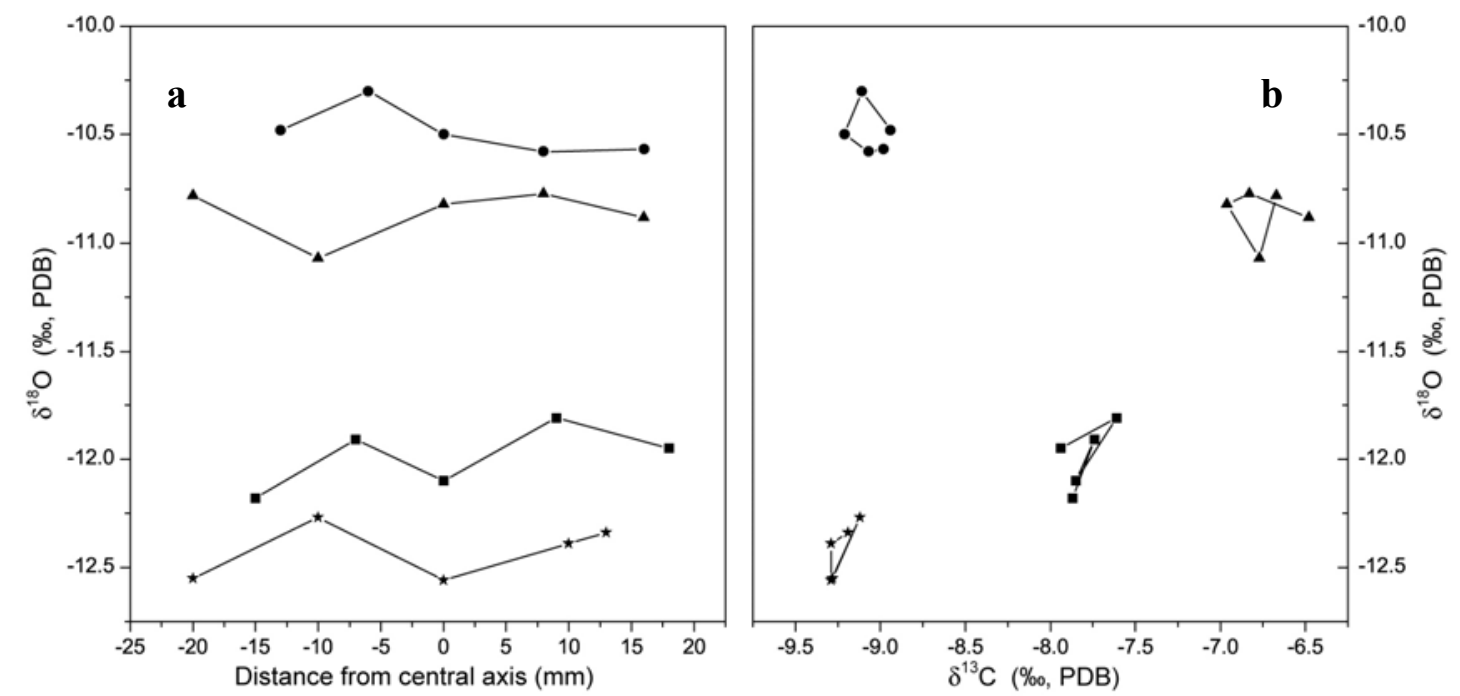

Figure DR5. $\quad \delta^{13} \mathrm{C}-\delta^{18} \mathrm{O}$ correlation for stalagmite XBL-1. The entire along-growth-axis data set shows a poor correlation between $\delta^{18} \mathrm{O}$ and $\delta^{13} \mathrm{C}\left(\mathrm{R}^{2}=\right.$ 0.032), indicating lack of kinetic fractionation effects during calcite precipitation (Hendy, 1971).

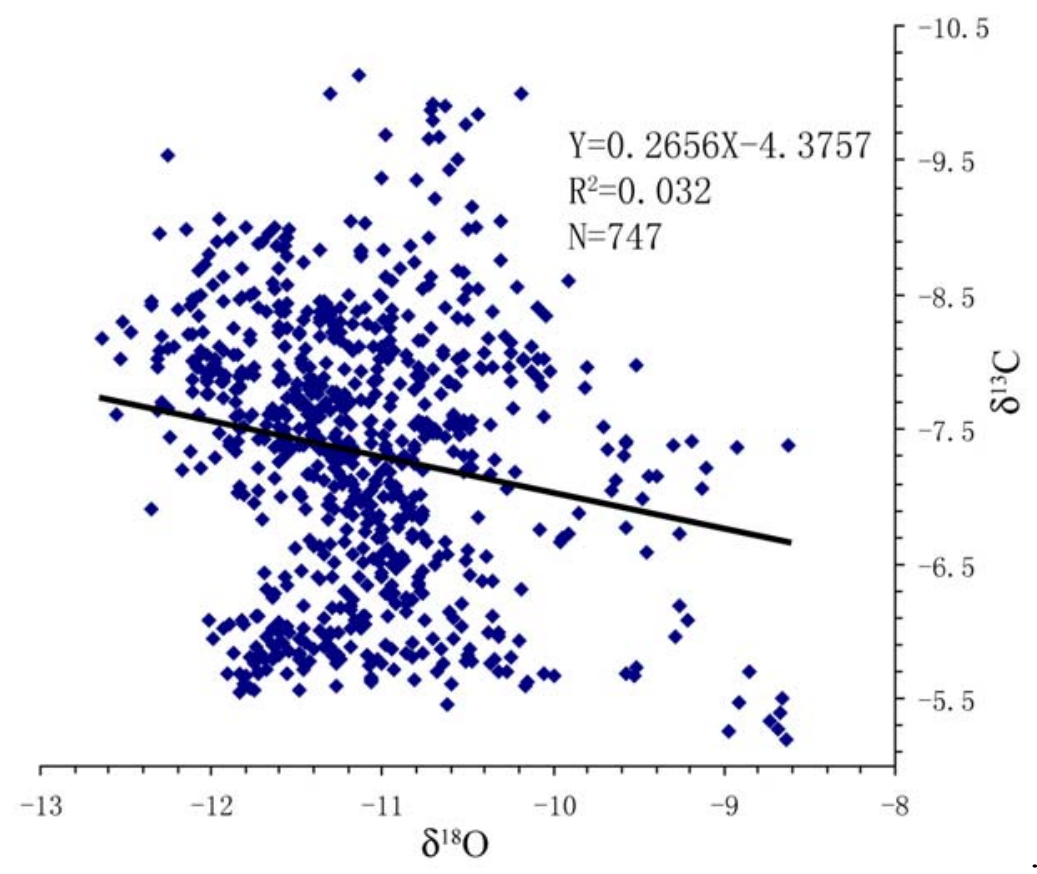


Table DR1 ${ }^{230}$ Th dating results of stalagmite XBL-1 from Xiaobailong Cave. The measurements were performed on a magnetic sector ICP-MS (Shen et al., 2002). Errors are $2 \sigma$. Decay constant values are: $\lambda_{230}=9.1577 \times 10^{-6} \mathrm{y}^{-1}, \lambda_{234}=2.8263 \times 10^{-6} \mathrm{y}^{-1}$ and $\lambda_{238}=1.55125 \times 10^{-10} \mathrm{y}^{-1}$ (Cheng et al., 2000). The ${ }^{230} \mathrm{Th}$ ages were corrected by an initial ${ }^{230} \mathrm{Th} /{ }^{232} \mathrm{Th}$ atomic ratio of $4.4 \pm 2.2 \times 10^{-6}$. Due to a very small amount of ${ }^{232} \mathrm{Th}$ incorporated in stalagmite XBL-1, the corrections are trivial ( $\leq 10$ years). Depths along the growth axis are relative to the top (youngest surface) of the stalagmite. yr BP: year before present (1950 AD).

\begin{tabular}{|c|c|c|c|c|c|c|c|c|}
\hline $\begin{array}{l}\text { Sample } \\
\text { Number }\end{array}$ & $\begin{array}{l}\text { Depth } \\
\text { (cm) }\end{array}$ & ${ }_{(p p b)}^{238} U$ & $\begin{array}{r}{ }^{232} \mathrm{Th} \\
\text { (ppt) }\end{array}$ & $\begin{array}{c}\delta^{234} U^{*} \\
\text { (measured) }\end{array}$ & $\begin{array}{l}{ }^{230} \mathrm{Th} /{ }^{238} \mathrm{U} \\
\text { (activity) }\end{array}$ & $\begin{array}{c}{ }^{230} \text { Th Age (yr BP) } \\
\text { (uncorrected) }\end{array}$ & $\begin{array}{c}{ }^{230} \text { Th Age (yr BP) } \\
\text { (corrected) }\end{array}$ & $\begin{array}{c}\delta^{234} U_{\text {Initial }} \\
\text { (corrected) }\end{array}$ \\
\hline MC-49 & 0.2 & $149.0 \pm 0.4$ & $508.5 \pm 4.1$ & $42.0 \pm 2.8$ & $0.1720 \pm 0.0016$ & $19631 \pm 215$ & $19536 \pm 219$ & $44.4 \pm 3.0$ \\
\hline JA-24 & 1.4 & $153.7 \pm 0.9$ & $9.1 \pm 3.4$ & $40.5 \pm 4.7$ & $0.2340 \pm 0.0020$ & $27719 \pm 303$ & $27717 \pm 303$ & $43.9 \pm 5.0$ \\
\hline JA-23 & 3.8 & $187.9 \pm 0.4$ & $3.0 \pm 3.0$ & $44.3 \pm 2.2$ & $0.2948 \pm 0.0017$ & $36073 \pm 259$ & $36073 \pm 259$ & $49.1 \pm 2.5$ \\
\hline JA-22 & 6.0 & $202.2 \pm 0.4$ & $1.8 \pm 3.4$ & $43.8 \pm 1.9$ & $0.2964 \pm 0.0012$ & $36342 \pm 188$ & $36341 \pm 188$ & $48.5 \pm 2.1$ \\
\hline XBL-1 & 7.5 & $197.7 \pm 0.3$ & $5.1 \pm 5.8$ & $42.3 \pm 1.6$ & $0.2956 \pm 0.0015$ & $36288 \pm 224$ & $36287 \pm 224$ & $46.9 \pm 1.8$ \\
\hline XBL-9 & 10.6 & $159.4 \pm 0.3$ & $283.0 \pm 12.9$ & $45.3 \pm 2.7$ & $0.2981 \pm 0.0025$ & $36517 \pm 383$ & $36468 \pm 384$ & $50.2 \pm 3.0$ \\
\hline XBL-2 & 13.3 & $146.0 \pm 0.2$ & $4.8 \pm 9.2$ & $44.9 \pm 2.5$ & $0.3087 \pm 0.0019$ & $38091 \pm 306$ & $38090 \pm 306$ & $50.0 \pm 2.8$ \\
\hline XBL-10 & 14.5 & $144.0 \pm 0.2$ & $310.8 \pm 11.1$ & $43.4 \pm 2.2$ & $0.3201 \pm 0.0024$ & $39852 \pm 383$ & $39792 \pm 384$ & $48.6 \pm 2.4$ \\
\hline XBL-3 & 17.3 & $241.6 \pm 0.3$ & $109.9 \pm 5.6$ & $43.5 \pm 1.7$ & $0.3224 \pm 0.0014$ & $40195 \pm 232$ & $40183 \pm 232$ & $48.7 \pm 1.9$ \\
\hline XBL-4 & 27.6 & $222.9 \pm 0.3$ & $664.3 \pm 4.2$ & $43.3 \pm 1.4$ & $0.3310 \pm 0.0013$ & $41501 \pm 207$ & $41418 \pm 211$ & $48.7 \pm 1.6$ \\
\hline XBL-5 & 38.5 & $230.9 \pm 0.3$ & $43.6 \pm 3.6$ & $46.0 \pm 1.2$ & $0.3375 \pm 0.0013$ & $42366 \pm 208$ & $42361 \pm 208$ & $51.8 \pm 1.3$ \\
\hline XBL-11 & 44.5 & $326.3 \pm 0.5$ & $287.3 \pm 6.4$ & $42.9 \pm 1.4$ & $0.3413 \pm 0.0015$ & $43113 \pm 243$ & $43088 \pm 243$ & $48.4 \pm 1.6$ \\
\hline XBL-6 & 45.2 & $353.7 \pm 0.4$ & $0.2 \pm 4.1$ & $43.1 \pm 1.1$ & $0.3404 \pm 0.0012$ & $42957 \pm 191$ & $42957 \pm 191$ & $48.7 \pm 1.2$ \\
\hline XBL-14 & 47 & $299.9 \pm 0.4$ & $77.7 \pm 7.5$ & $42.5 \pm 1.7$ & $0.3437 \pm 0.0018$ & $43495 \pm 289$ & $43488 \pm 289$ & $48.1 \pm 1.9$ \\
\hline XBL-7 & 47.3 & $696.7 \pm 0.9$ & $36.7 \pm 6.2$ & $42.4 \pm 1.1$ & $0.3454 \pm 0.0011$ & $43770 \pm 185$ & $43768 \pm 185$ & $48.0 \pm 1.2$ \\
\hline XBL-12 & 47.75 & $378.0 \pm 0.5$ & $175.6 \pm 3.2$ & $42.3 \pm 1.2$ & $0.3492 \pm 0.0015$ & $44365 \pm 237$ & $44355 \pm 237$ & $48.0 \pm 1.2$ \\
\hline XBL-17 & 49.1 & $844.2 \pm 1.6$ & $187.6 \pm 4.5$ & $42.6 \pm 1.8$ & $0.3552 \pm 0.0016$ & $45350 \pm 268$ & $45344 \pm 268$ & $48.4 \pm 2.0$ \\
\hline XBL-8 & 50.5 & $257.6 \pm 0.3$ & $26.6 \pm 4.8$ & $40.0 \pm 1.2$ & $0.3630 \pm 0.0014$ & $46686 \pm 233$ & $46683 \pm 233$ & $45.7 \pm 1.4$ \\
\hline XBL-16 & 52.2 & $6927.8 \pm 24.9$ & $390.4 \pm 15.1$ & $32.7 \pm 1.6$ & $0.3756 \pm 0.0016$ & $49175 \pm 290$ & $49173 \pm 290$ & $37.6 \pm 1.8$ \\
\hline XBL-15 & 53.7 & $4593.9 \pm 15.5$ & $804.2 \pm 7.4$ & $30.9 \pm 1.6$ & $0.3802 \pm 0.0016$ & $50073 \pm 286$ & $50068 \pm 286$ & $35.6 \pm 1.8$ \\
\hline$J A-19$ & 54.9 & $7409.5 \pm 36.6$ & $892.6 \pm 7.5$ & $34.7 \pm 2.2$ & $0.3882 \pm 0.0022$ & $51141 \pm 400$ & $51137 \pm 400$ & $40.1 \pm 2.5$ \\
\hline$J A-20$ & 58.75 & $7268.1 \pm 23.6$ & $5750.7 \pm 19.7$ & $33.8 \pm 1.5$ & $0.3960 \pm 0.0018$ & $52527 \pm 326$ & $52504 \pm 326$ & $39.2 \pm 1.8$ \\
\hline
\end{tabular}

